\title{
In memoriam Jean-Marie Tschopp (1948-2020)
}

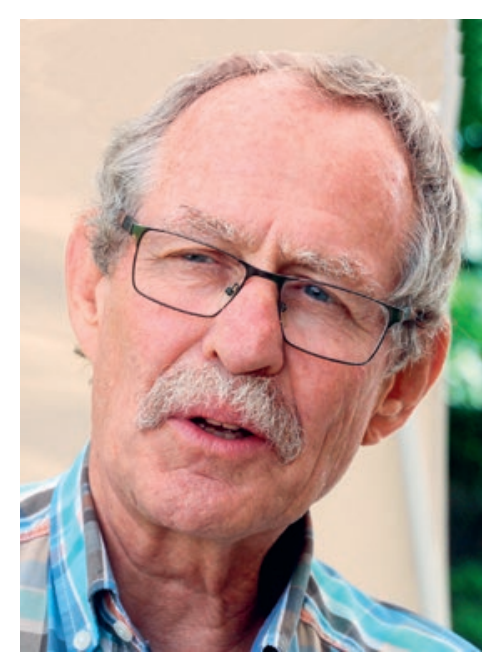

Le 20 septembre 2020, Jean-Marie Tschopp nous a quittés, décédé des suites d'un accident survenu alors qu'il se rendait de Montana à Genève à vélo $(180 \mathrm{~km})$. Il était âgé de 72 ans. Toute sa vie, il est allé de l'avant, non pas de manière téméraire, mais obstinée, convaincu que lorsqu'on a une bonne idée il faut y mettre toute son énergie, ne pas reculer devant les obstacles et entrầner les autres avec soi. Il a apporté une contribution majeure à la pneumologie suisse et internationale, tant par ses publications que par sa personnalité et son enthousiasme.

Après une jeunesse valaisanne, il fait à Genève ses études de médecine et sa spécialisation en médecine interne, puis en pneumologie. Il dira avoir été profondément marqué par ses «maîtres», ceux qui lui ont appris son métier: Jean Fabre (Policlinique de Médecine), Alain Junod et Rodolphe de Haller (Pneumologie), John Batten (Brompton Hospital, Londres) ou Christian Boutin (Marseille). En 1985, il est nommé médecindirecteur du Centre Valaisan de Pneumologie à Montana et en 2003 Professeur associé à la Faculté de Médecine de Genève. De 1991 à 2013, il sera co-requérant de l'étude de cohorte suisse sur les effets de la pollution atmosphérique sur la santé (SAPALDIA) où son activité sera essentielle tant sur le plan scientifique qu'organisationnel. Il sera aussi l'un des premiers à faire collaborer médecins, pharmaciennes et pharmaciens, physiothérapeutes, infirmières et infirmiers dans un projet original d'éducation thérapeutique pour l'asthme qui fera figure de modèle en Suisse. Sur le plan international, il deviendra un des membres les plus actifs du groupe de travail sur la thoracoscopie de l'European Respiratory Society. Parallèlement, avec son équipe, il fera du Centre Valaisan de Pneumologie un service moderne. Il introduit l'oxygénothérapie à domicile grâce à la Ligue pulmonaire valaisanne et crée le premier laboratoire du sommeil du Valais. Il participera à la mise en place des nouvelles structures hospitalières de son canton (chef du Département de médecine du Centre Hospitalier du Centre du Valais). Cette énumération n'est de loin pas exhaustive, mais témoigne de l'élan extraordinaire que Jean-Marie Tschopp a su créer autour de lui. Il n'hésitait pas à bousculer pour faire avancer, mais c'était avec l'objectif de promouvoir une médecine où prévaut la relation humaine et qui place le patient au centre de nos préoccupations.

Valaisan de toutes ses fibres, il était entier, fonceur, généreux, débordant d'énergie, toujours prêt à donner un coup de main, avec cette simplicité qui le rendait attachant. Il a grandement contribué au succès des congrès «Quadrimed» que les quatre cliniques d'altitude de Montana (Bernoise, Lucernoise, Genevoise et Valaisanne) organisent annuellement pour les médecins généralistes. Il tenait beaucoup à une formation efficiente et pragmatique des médecins de premiers recours.

Amoureux des balades en montagne, il entraîna ses collaborateurs et même ses patients à de nombreuses reprises vers les hauteurs pour valoriser l'effort physique. Par ses qualités humaines et celles de sa femme, Maria Pia, s'est créé un réseau impressionnant, où le professionnel et l'amical ne se distinguaient guère. Combien de réunions de travail se terminaient dans la convivialité et la bonne humeur - et combien de verres amicaux venaient conclure une discussion approfondie autour d'une meilleure médecine!

La disparition brutale de Jean-Marie Tschopp laisse ses proches, ses amis et ses anciens collègues dans la tristesse et la consternation. Il laissera derrière lui une image marquante de la pneumologie en Suisse. Nous adressons toute notre sympathie à son épouse et à leurs trois filles, ainsi qu'à toute sa famille.

Professeur Thierry Rochat, Dr Rainer Kaelin, Dr Jean Georges Frey 